\title{
ANÁLISIS DE LA SOLUBILIDAD DE LA MEZCLA GASOLINA-ETANOL-AGUA A DIFERENTES PRESIONES Y TEMPERATURAS
}

\section{SOLUBILITY ANALYSIS OF GASOLINE-ETHANOL-WATER MIXTURES AT DIFFERENT PRESSURES AND TEMPERATURES}

Michael Fernando Rondón', Juan Miguel Mantilla González², Alejandro Muñoz Rodríguez³

Fecha de recepción: 25 de Septiembre de 2014

Fecha de aprobación: 15 de Diciembre de 2014

Referencia: M.F. Rondón, J.M. Mantilla González, A. Muñoz Rodríguez (2015). Análisis de la solubilidad de la mezcla gasolina-etanol-agua a diferentes presiones y temperaturas. Ciencia e Ingeniería Neogranadina, 25 (1), pp. 61 - 75

\section{RESUMEN}

En este artículo se presenta el efecto de pruebas de solubilidad hechas a mezclas gasolinaetanol-agua mediante ensayos de tolerancia al agua (temperatura de separación de fase) para muestras de E10, E15, E20, E25 y E30. Adicionalmente, se estudia el efecto de la presión de vacío sobre la separación de la mezcla. Los resultados muestran que las temperaturas de separación dependen inversamente de la cantidad de etanol en la mezcla combustible y directamente de la cantidad de agua en la misma. Así mismo, se encontró que la influencia de la presión de vacío es mínima cuando la mezcla combustible se encuentra en estado líquido.

Palabras clave: Mezcla gasolina-etanol, tolerancia al agua, temperatura de separación de fase, efecto de presiones de vacío, contenido de agua, solubilidad.

1. Ingeniero Mecánico, Grupo de Modelado y Métodos Numéricos en Ingeniería GNUM. Universidad Nacional de Colombia, Bogotá, Colombia, mfrondona@unal.edu.co.

2. Ingeniero Mecánico, Ph.D., Profesor Asociado, Grupo de Modelado y Métodos Numéricos en Ingeniería GNUM. Universidad Nacional de Colombia, Bogotá, Colombia, jmmantillag@unal.edu.co

3. Ingeniero Mecánico, M.Sc. en Ingeniería Mecánica, Profesor Tiempo Completo, Grupo de Investigación en Energía y Eficiencia GIEE. Universidad Central, Bogotá, Colombia, amunozr3@ucentral.edu.co 


\section{ABSTRACT}

This work presents the effect of solubility tests made to gasoline-ethanol-water mixtures. In particular, water tolerance tests (phase separation temperature) applied on E10, E15, E20, E25 and E30 blends. In addition, the vacuum pressure effect on mixture separation is analyzed. Results showed that separation temperatures depend indirectly on ethanol concentration within the mixture and directly on water concentration. Likewise, it was found that vacuum pressure influence is negligible when the fuel blend is under liquid state.

Keywords: Gasoline-ethanol blend, water tolerance, phase separation temperature, vacuum pressure effect, water content, solubility.

\section{INTRODUCCIÓN}

La preocupación mundial por la escasez de los derivados del petróleo y los efectos ambientales que implica el uso de estos combustibles han llevado a los gobiernos a encontrar e implementar soluciones que garanticen una menor dependencia de los mismos en pro de la protección ambiental. El uso de etanol como combustible alternativo para los motores a gasolina ha sido una de las soluciones más populares y a la vez más controversiales. El etanol puede producirse por varios métodos. Sin embargo, el más usado es el proceso de fermentación de azúcares. A nivel mundial, la producción de etanol proviene de la caña de azúcar y del maíz. La primera materia prima ha sido altamente usada en Brasil y recientemente en Colombia, mientras que el maíz se ha utilizado en Estados Unidos [1].

Indistintamente del método de producción, el etanol presenta un serio inconveniente para su producción, manejo, distribución, almacenamiento yutilizacióncomocombustible en motores: la presencia de agua. El etanol es altamente afín al agua [2]. La presencia de agua en la mezcla etanol-gasolina trae consigo los siguientes efectos sobre los motores: (i) corrosión sobre los componentes internos del sistema de inyección y sus sistemas asociados y (ii) alteración en el funcionamiento de los motores debido a la separación de la mezcla.

El mecanismo por el cual la corrosión de los componentes metálicos del sistema de inyección de combustible ocurre es parcialmente debido al incremento de la cantidad de agua en el combustible y los ácidos orgánicos presentes en los oxigenantes comerciales [3]. Existe una concentración específica en la cual el agua contenida dentro de la mezcla no puede mantenerse unida a la misma y termina separándose totalmente [4-5]. Esta separación del agua de la mezcla gasolina-etanol aumenta en forma notoria sus impactos adversos sobre los componentes internos. La corrosión galvánica, que ocurre en presencia de campos eléctricos, es altamente promovida por la presencia de etanol-agua en los combustibles, ya que el etanol es un mejor conductor que la gasolina [6-7]. El etanol en presencia del agua es la causa de la degradación de los materiales plásticos y elastómeros de los sistemas de inyección y la causa más frecuente de la obstrucción de 
filtros y ductos de manejo de combustible en los motores operados con este tipo de mezclas [8-9]. En referencia a los impactos sobre el funcionamiento del motor cuando son operados con mezclas de etanol-gasolinaagua, la manejabilidad del vehículo puede verse seriamente afectada con la presencia de esta mezcla, sobre todo cuando se requiere alcanzar máxima potencia. El deterioro de la operación del vehículo dependerá del año de fabricación del mismo y la tecnología usada en su sistema de alimentación. Aunque motores alimentados con carburador y con inyección se ven impactados en forma diferente, en general, el consumo de combustible y las emisiones evaporativas se aumentan, y los problemas de arranque en caliente se incrementan [10-12].

En Colombia se está utilizando etanol producido a partir de la caña de azúcar desde el año 2006. Progresivamente se ha implementado en todo el país, exceptuando regiones fronterizas, garantizando un $8 \%$ en volumen de etanol en la gasolina [13]. Además de esto, en el decreto 4892 de 2011 del Ministerio de Minas y Energía se incentiva el uso de mezclas gasolina y etanol, este último con proporción mayor al 10\% en volumen. De hecho, en pruebas realizadas por la Universidad Tecnológica de Pereira se encontró que para los vehículos seleccionados no hay efectos adversos con el uso de gasolina con $20 \%$ de etanol [14]. Adicionalmente, en Colombia, y en el caso particular de Bogotá, cerca del $43 \%$ de los vehículos automotores que utilizan gasolina como combustible tienen un sistema de alimentación con carburador [15]. Esto crea un parque automotor con una diversidad de tecnologías que permite plantear, junto con lo mostrado anteriormente, que la posibilidad de que el combustible se mezcle con agua, en especial al aumentar la cantidad de etanol, es real y se presenta como un tema importante para estudiar a fondo.
En particular, existen cinco estudios previos que han definido las variables que afectan el comportamiento de la mezcla y pueden causar su separación. Estas variables son: cantidad de etanol, cantidad de agua, composición de la gasolina (aromáticos y aditivos) y temperatura.

Entre los estudios que corroboran las variables anteriormente mencionadas como las determinantes de la solubilidad de la mezcla se encuentra el estudio de Strus et al. [16], que muestra cómo el contenido de sustancias hidrófilas como el etanol, el metanol, el propanol, el metil-propanol, el butano y el MTBE aumentan la tolerancia al agua de la gasolina, cada uno de manera diferente pero todos en forma proporcional a su cantidad (Figura 1).

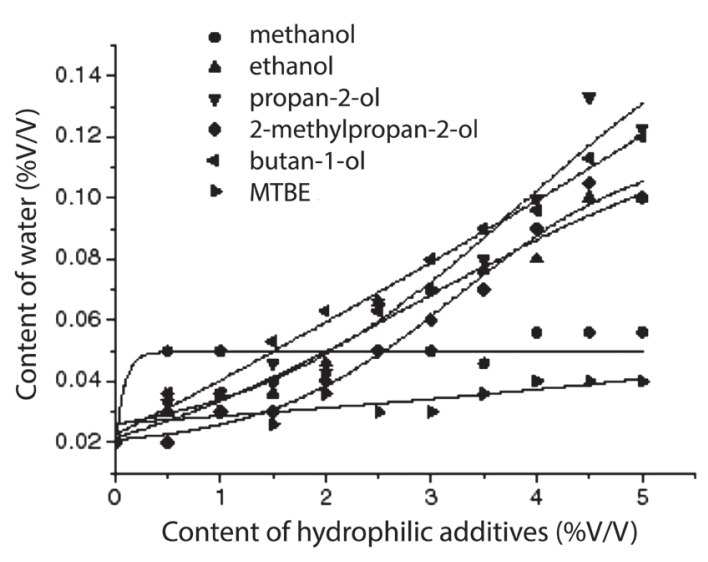

Figura 1. Solubilidad del agua en gasolina. Fuente: Strus et al., 2007.

Otro estudio es el de Torres et al. [17] en el que se presenta la solubilidad de la mezcla a diferentes temperaturas (entre $273 \mathrm{~K}$ y $315 \mathrm{~K})$. Adicionalmente, y corroborando lo anteriormente mencionado, presenta a la cantidad de etanol como variable en mezclas 
E5, E10 y E15. La temperatura afecta el comportamiento de la mezcla de manera que a menor temperatura menor tolerancia al agua. A mayor temperatura, la energía de interacción entre las moléculas de la gasolina, el etanol y el agua aumenta, produciendo mayor contacto entre éstas y, por ende, elevando el grado de solubilidad

Kato [18] analizó el contenido de agua en las mezclas a diferentes temperaturas y desarrolló un diagrama de tolerancia al agua para bajos contenidos de etanol. La Figura 2 muestra cómo al aumentar el contenido de agua en la mezcla etanol-gasolina, la temperatura de separación de la misma se alcanza a mayores valores.

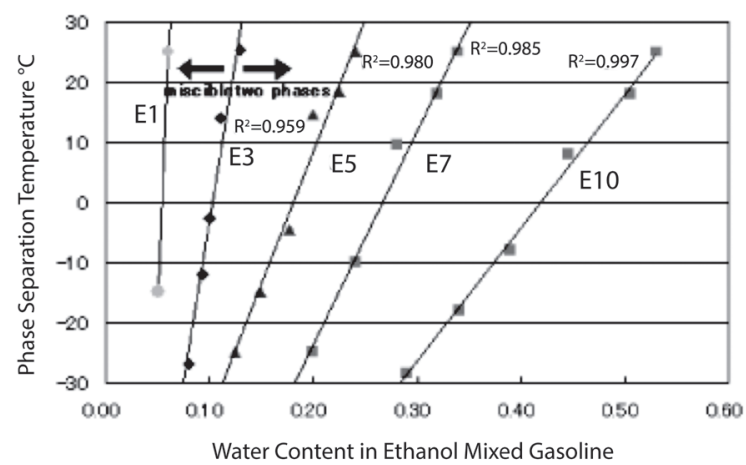

Figura 2. Tolerancia al agua de la mezcla gasolinaetanol-agua.

Fuente: Kato, 2006

Otro estudio que reporta la separación de la mezcla en función de la temperatura es el de Reynolds [19], presentado en la Figura 3. Se pueden observar los diferentes límites del contenido de agua previos a la separación de fase para mezclas de $5.7 \%, 7.7 \%$ y $10 \%$ de contenido de etanol en volumen.
Karaosmanoglu et al. [20] estudiaron cuatro diferentes composiciones de gasolina como se muestra en la Tabla 1. Los contenidos de aromáticos varían desde 27.20 para la gasolina denominada G1 hasta 59.94 para la G2. Estas gasolinas son mezcladas con 5 , 10,15 , y $20 \%$ por volumen de etanol anhidro y llamadas B1, B2, B3, y B4 respectivamente. La tolerancia al agua es mostrada en la Tabla 2. Es evidente cómo, a mayores cantidades de aromáticos o mayores cantidades de etanol, la temperatura límite para la separación de fase es menor. En otras palabras, la tolerancia al agua aumenta con el contenido de aromáticos y de etanol.

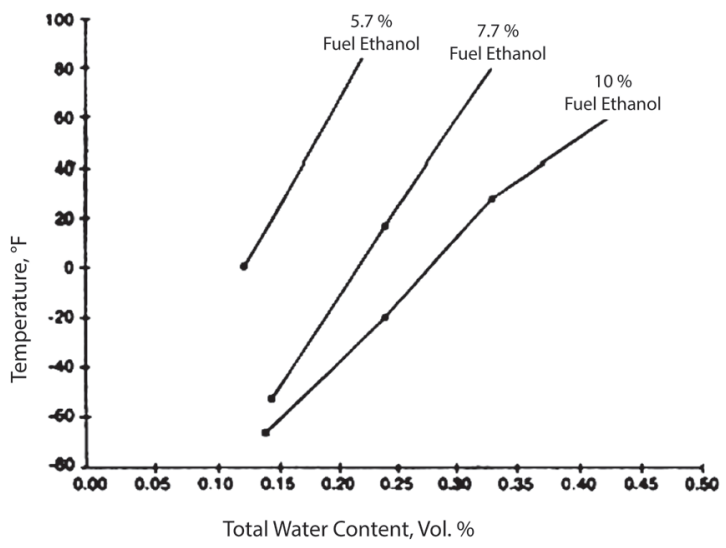

Figura 3. Tolerancia al agua de la mezcla gasolina etanol para contenidos de etanol de 5.7, 7.7 y $10 \%$ en volumen.

Fuente: Reynolds, 2002

La siguiente variable en la solubilidad de la mezcla es la composición de la gasolina base. La solubilidad se incrementa con el aumento de la concentración de aromáticos en la mezcla etanol-gasolina. Es por esto que la mezcla etanol-gasolina extra con mayor concentración de estos compuestos tolera más agua que 
Tabla 1. Especificaciones de cuatro diferentes tipos de gasolinas.

\begin{tabular}{|c|c|c|c|c|c|}
\hline \multicolumn{6}{|c|}{ Gasolina } \\
\hline Propiedad & Método de ensayo ASTM & G1 & G2 & G3 & G4 \\
\hline Color & D1298 & Amarillo & Amarillo & Amarillo & Amarillo \\
\hline Densidad @15C (kg/m3) & D1319 & 734.6 & 742.1 & 762.5 & 795.2 \\
\hline \multicolumn{6}{|c|}{ Composición (peso\%) } \\
\hline \multicolumn{2}{|c|}{ Hidrocarburos saturados } & 72.20 & 60.01 & 49.70 & 38.96 \\
\hline \multicolumn{2}{|c|}{ Hidrocarburos aromáticos } & 27.20 & 39.02 & 48.80 & 59.94 \\
\hline \multicolumn{2}{|c|}{ Hidrocarburos olefínicos } & 0.60 & 0.97 & 1.50 & 1.10 \\
\hline Agua (\%v/v) & D1744 & 0.1227 & 0.3700 & 0.2504 & 0.1508 \\
\hline Azufre (kg/m3) & D381 & $28 \times 10^{-3}$ & $8 \times 10^{-3}$ & $4 \times 10^{-3}$ & $6 \times 10^{-3}$ \\
\hline Ensayo de destilación & D86 & & & & \\
\hline \multicolumn{2}{|c|}{ Destilado $10 \%$} & 57 & 50 & 52 & 78 \\
\hline \multicolumn{2}{|c|}{ Destilado $50 \%$} & 99 & 89 & 121 & 132 \\
\hline \multicolumn{2}{|c|}{ Destilado $90 \%$} & 155 & 143 & 159 & 162 \\
\hline \multicolumn{2}{|c|}{ Residuo (\%) } & 1.0 & 0.5 & 0.5 & 0.5 \\
\hline \multicolumn{2}{|c|}{ Pérdida (\%) } & 1.0 & 0.5 & 1.0 & 1.0 \\
\hline Presión de vapor Reid & D323 & 56.7 & 63.8 & 77.0 & 48.6 \\
\hline \multicolumn{6}{|l|}{ Número de octano } \\
\hline Método de investigación & D2699 & 82.0 & 85.0 & 96.6 & 97.5 \\
\hline Método motor & D2700 & 76.0 & 74.5 & 86.5 & 86.6 \\
\hline Corrosión en cobre $50^{\circ} \mathrm{C} 3 \mathrm{~h}$ & D130 & No 1a & No 1a & No 1a & No 1a \\
\hline
\end{tabular}

Fuente: Karaosmanoglu et al., 1996.

la gasolina regular para una determinada temperatura y porcentaje de etanol; esto se debe a la afinidad de estos compuestos con el agua [11].

La influencia de los aromáticos en función de la tolerancia al agua de la mezcla puede observarse en la Figura 4 para las mezclas
E5, E10 y E15. Con diferentes cantidades de aromáticos se encontró que el aumento de los mismos en la mezcla disminuye sensiblemente el punto de separación. Obsérvese que en la Figura 4 se indica el Cloud Point como nivel de tolerancia. Este se define como la temperatura en la que la cristalización inicial o separación de la fase del combustible comienza [21]. 


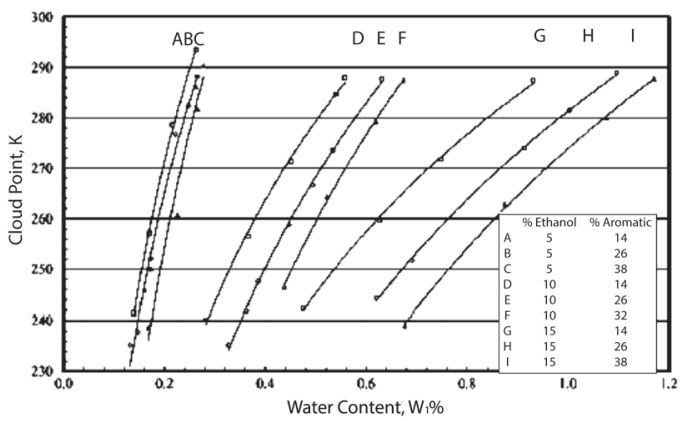

Figura 4. Tolerancia al agua de la mezcla gasolina-etanol en función de la cantidad de agua y de aromáticos.

Fuente: French et al., 2005.

Teniendo en cuenta lo anterior, se esboza en este trabajo una metodología experimental que permite verificar la solubilidad del etanol en la gasolina en presencia de agua cuando se cambia la temperatura y la presión a la que se somete la mezcla.

\section{MATERIALES Y MÉTODOS}

\subsection{PROCEDIMIENTO EXPERIMENTAL}

El objetivo de este estudio consistió en encontrar la influencia de las presiones de vacío en la separación de la mezcla gasolina etanol, en estado líquido, así como estudiar este efecto sobre algunas proporciones de etanol diferentes a las encontradas en la literatura.

El diseño del experimento se hizo basado en el procedimiento estandarizado por la

Tabla 2 Temperaturas de separación de fase (Phase Separation Temperatures, PST) para mezclas etanol-gasolina preparadas con gasolinas de composiciones diferentes.

\begin{tabular}{|c|c|c|c|c|c|c|}
\hline \multirow{2}{*}{ FOF \% } & \multirow{2}{*}{ EtOH \% } & \multirow{2}{*}{ H2O $\%$} & \multicolumn{5}{|c|}{ PST ${ }^{\circ} \mathrm{C}$} \\
\cline { 5 - 7 } & & & B1 & B2 & B3 & B4 \\
\hline 0 & 5 & 0.3 & $>39$ & $>39$ & $>39$ & $>39$ \\
\hline & 10 & 0.5 & 19.0 & 17.5 & 16.5 & 15.0 \\
\hline & 15 & 0.7 & 11.5 & 6.5 & 1.5 & -1.2 \\
\hline & 20 & 0.9 & 9.0 & 2.5 & -8.0 & -12.5 \\
\hline 1 & 5 & 0.3 & 16.5 & 12.5 & 10.5 & 8.5 \\
\hline & 10 & 0.5 & 5.5 & 4.5 & 3.0 & 2.5 \\
\hline & 15 & 0.7 & -1.0 & -3.0 & -8.0 & -12.5 \\
\hline & 20 & 0.9 & -3.0 & -8.0 & -13.5 & -18.2 \\
\hline 3 & 5 & 0.3 & -12.5 & -16.0 & -19.5 & -22.0 \\
\hline & 10 & 0.5 & -17.5 & -23.5 & -27.0 & -35.7 \\
\hline & 15 & 0.7 & -22.0 & -28.5 & -33.5 & -37.5 \\
\hline & 20 & 0.8 & -26.5 & -34.5 & -37.5 & -40.5 \\
\hline
\end{tabular}

Fuente: Karaosmanoglu et al, 1996 
norma ASTM D6422-99 [22], con algunas modificaciones realizadas para poder medir la influencia de la presión.

A diferencia de estudios previos en los que la temperatura de la mezcla permanecía constante y se agregaba agua a la mezcla hasta encontrar la turbidez, en este estudio la composición de agua de la mezcla se mantuvo invariable mientras la temperatura era modificada usando un baño de enfriamiento.

\subsubsection{Caracterización de la gasolina}

Se utilizó gasolina denominada corriente (bajo octanaje) sin mezclar. La gasolina cumple la norma local de calidad exigida por el ente normativo nacional ICONTEC NTC1380 [23]. La gravedad específica fue determinada mediante el ensayo ASTM D1298 [24], y es igual a 0.739. La destilación de la gasolina se llevó a cabo por el método ASTM D86 [24]. Los resultados de la curva de destilación son presentados en la Tabla 3.

El contenido de agua es determinado mediante el procedimiento ASTM D1744 [25]. Los resultados de esta prueba para el etanol y la gasolina fueron respectivamente $0.060 \mathrm{y}$ $0.001 \%$.

\subsubsection{Montaje experimental}

El montaje se constituye principalmente en un contenedor de muestra con una marca de control que señala $40 \mathrm{ml}$. Una vez llenado con la mezcla que se desea probar es cerrado con un tapón de caucho que aloja un termómetro ubicado en su centro y una manguera conectada a un vacuómetro, así como una aguja que unida a una jeringa es la encargada de producir presiones de vacío (presiones menores a la presión atmosférica).
El contenedor es depositado en una camisa de vidrio, aislado de ésta mediante bandas de caucho y un disco de fieltro. Este montaje experimental se muestra en la Figura 5. La camisa es, a su vez, depositada en un baño de enfriamiento consistente en un recipiente de poliestireno que es llenado con hielo seco, como se muestra en la Figura 6.

Tabla 3 Resultados curva de destilación a la gasolina usada siguiendo procedimiento ASTM D86.

\begin{tabular}{|c|c|c|}
\hline & Prueba I & Prueba II \\
\hline \% Destilado & \multicolumn{2}{|c|}{ Temperatura $\left({ }^{\circ} \mathrm{C}\right)$} \\
\hline Primera Gota & 31 & 34 \\
\hline 10 & 54 & 54 \\
\hline 20 & 58 & 61 \\
\hline 30 & 61 & 77 \\
\hline 40 & 76 & 80 \\
\hline 50 & 91 & 89 \\
\hline 60 & 103 & 103 \\
\hline 70 & 126 & 123 \\
\hline 80 & 142 & 144 \\
\hline 90 & 170 & 171 \\
\hline $\begin{array}{c}\text { Máxima } \\
\text { Temperatura }\end{array}$ & 192 con 95\% & $196 \operatorname{con} 95 \%$ \\
\hline Volumen destilado & $96 \%$ & $96 \%$ \\
\hline Residuo & $1.60 \%$ & $1.80 \%$ \\
\hline
\end{tabular}

\subsubsection{Procedimiento}

La mezcla fue preparada en el Laboratorio de Combustibles de la Facultad de Ingeniería de la Universidad Nacional de Colombia, sede Bogotá, bajo atmósfera controlada y verificando los contenidos exactos de gasolina, etanol y agua. 
Cada mezcla se depositó en el contenedor y selló con el tapón de caucho, previo ajuste del baño de enfriamiento. Para el caso de las muestras probadas en condiciones de vacío, este fue producido utilizando la jeringa. En caso contrario, la aguja fue cubierta de manera tal que a través de ella no saliera mezcla.

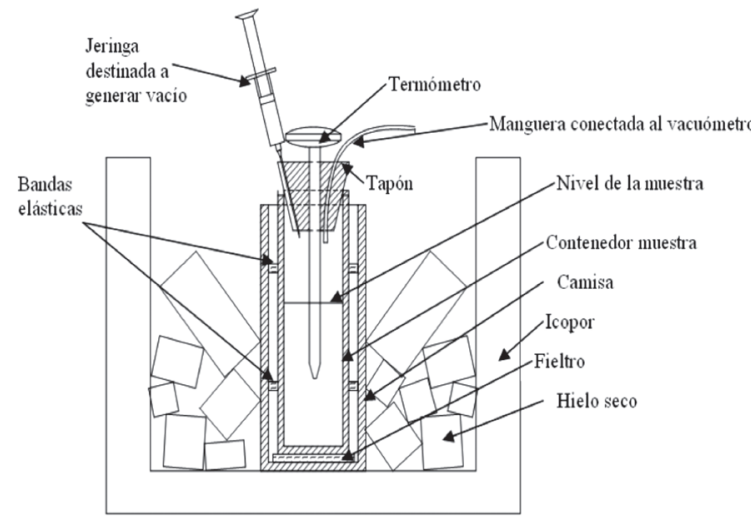

Figura 5. Montaje experimental.

Las presiones alcanzadas por el sistema de vacío construido fueron de $-26 \mathrm{cmHg}$, que para las condiciones de Bogotá representan 492 $\mathrm{mmHg}$ absolutos (65 kPa).

Posteriormente, el contenedor con la muestra es ubicado en la camisa. Cuando la temperatura llega a $14^{\circ} \mathrm{C}$, el contenedor es retirado de la camisa, agitado y limpiado mediante un paño empapado con etanol. Seguidamente se verifica si se presenta o no separación en la muestra. En caso de no presentarse separación, el contenedor es devuelto a la camisa.

Concada $2{ }^{\circ} \mathrm{C}$ de disminución de la temperatura, el contenedor es retirado, agitado y limpiado. Una vez encontrada la temperatura en la cual se observa la separación, esta es reportada y el contenedor con la muestra son expuestos a la temperatura ambiente.

Por cada aumento de $2^{\circ} \mathrm{C}$ de la temperatura de la muestra expuesta a la temperatura ambiente, esta es agitada y revisada hasta encontrar la temperatura en la cual la mezcla se recombina. Esta temperatura es registrada y la
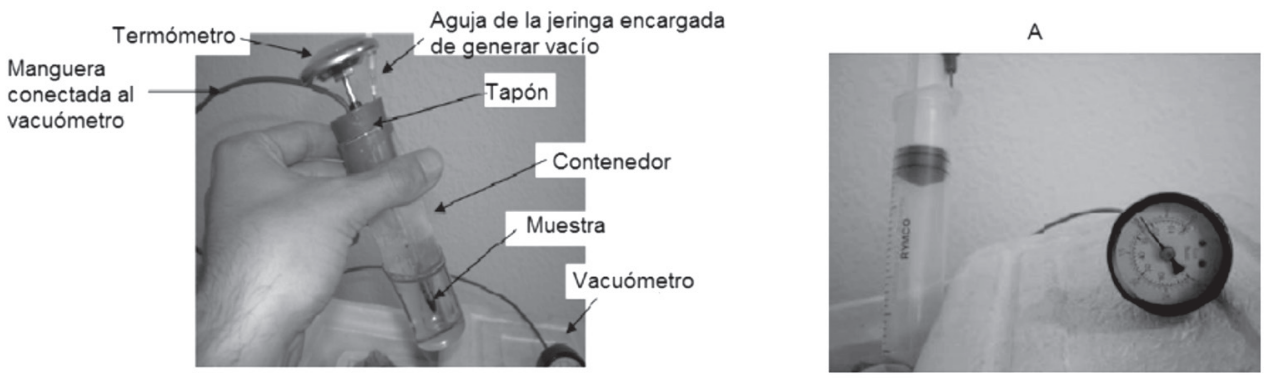

B
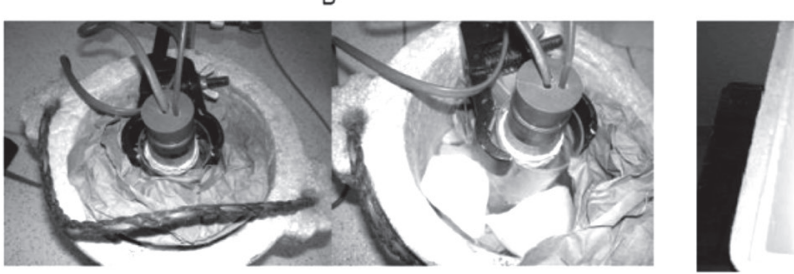

C

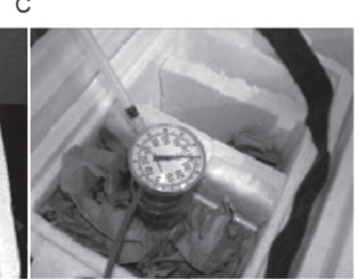

Figura 6. a) Detalle del montaje, b) Generación de vacío, c) Preparación del baño de enfriamiento. 
temperatura promedio, entre la de separación y la de recombinación, es la temperatura definida como «temperatura de separación de fase».

\subsubsection{Preparación de las mezclas}

Se realizaron pruebas con mezclas E10, E15, E20, E25 y E30. De cada mezcla se tomaron muestras de $40 \mathrm{ml}$. Se mezclaron $120 \mathrm{ml}$ con la cantidad de etanol y agua deseada por cada muestra, de manera que la preparación de las mezclas fuera sencilla y con menores posibilidades de error al medir el contenido de agua a agregar. Esta mezcla se almacenó en recipientes que se mantuvieron cerrados para evitar el contacto con la atmósfera.

Las mezclas se realizaron depositando cantidades de gasolina en un vaso de precipitados del cual, mediante jeringas, se tomó el volumen deseado para ser depositado en el frasco contenedor de la mezcla. La gasolina no utilizada del contenedor se volvió a depositar con el resto de la gasolina sin etanol, considerando que esta gasolina, al ser libre de etanol, no absorbería agua de la atmósfera.

Un procedimiento similar se realizó con el etanol, salvo que el etanol no utilizado no se devolvió sino que se depositó en otros contenedores y fue usado para limpiar el contenedor de la prueba, de manera que se permitiera la observación de la separación o no de la mezcla. A la mezcla se le agregó el agua mediante una jeringa de $1 \mathrm{ml}$. De esta mezcla se tomaron muestras de $40 \mathrm{ml}$ en el contenedor con las que se trabajó en el experimento. La mezcla que no fue utilizada se almacenó en el frasco contenedor debidamente cerrado para disminuir el contacto con la atmósfera.

Se decide evaluar las mezclas E10, E15, E20, E25 y E30 con bajas cantidades de agua, dentro del rango de 3000 a 5000 ppm. Como se explicará más adelante, este margen de cantidad de agua resulta muy bajo para las mezclas E30 y, por esta razón, se decidió implementar mezclas con 6000 a 8000 ppm.

\section{RESULTADOS Y DISCUSIÓN}

\subsection{Temperaturas de separación}

Los resultados obtenidos para las muestras analizadas a la presión atmosférica de Bogotá, $75 \mathrm{kPa}$, se observan en la Tabla 4.

Un análisis más acorde debe hacerse con la temperatura inicial en vez de la temperatura de separación de fase que fue definida previamente. Una de las razones para hacer el análisis basado en la temperatura inicial es que la temperatura de recombinación parece más dependiente de la agitación que se le realice a la muestra que de la temperatura misma. La segunda razón es que muchos de los estudios realizados reportan la temperatura inicial y, con el objetivo de poder comparar estos resultados con los obtenidos por otros investigadores, se hace necesario reportar los resultados de las temperaturas iniciales.

En la misma forma, como varios investigadores han encontrado en estudios previos [16-1719], las mezclas con bajas cantidades de etanol son más propensas a la separación porque soportan menor cantidad de agua. Es así como el E10 se separa con concentraciones mayores o iguales a 4500 ppm.

De la misma forma, concentraciones altas de etanol resultan bastante tolerantes al agua. Muestras con $30 \%$ de etanol en volumen no presentan separación para los rangos de concentración de agua menores a 6000 ppm y 
Tabla 4 Temperaturas de separación de fase (C) a presión ambiente (75kPa).

\begin{tabular}{|c|c|c|c|c|c|}
\hline $\begin{array}{l}\text { Agua } \\
\text { (ppm) }\end{array}$ & E10 & E15 & E20 & E25 & E30 \\
\hline 3000 & -16 & $\begin{array}{c}\text { No se } \\
\text { encontró }\end{array}$ & \multicolumn{2}{|c|}{$\begin{array}{c}\text { No se } \\
\text { encontró }\end{array}$} & \\
\hline 3500 & -8 & -34 & \multicolumn{2}{|c|}{$\begin{array}{l}\text { No se } \\
\text { encontró }\end{array}$} & \\
\hline 4000 & -6 & -17 & -40 & $\begin{array}{c}\text { No se } \\
\text { encontró }\end{array}$ & \\
\hline 4500 & $\begin{array}{l}\text { Separación } \\
\text { inmediata }\end{array}$ & -16 & -38 & & \\
\hline 5000 & $\begin{array}{c}\text { Separación } \\
\text { inmediata }\end{array}$ & -20 & -34 & $\begin{array}{c}\text { No se } \\
\text { encontró }\end{array}$ & $\begin{array}{c}\text { No se } \\
\text { encontró }\end{array}$ \\
\hline 6000 & & & & & $\begin{array}{c}\text { No se } \\
\text { encontró }\end{array}$ \\
\hline 7000 & & & & & -38 \\
\hline 8000 & & & & & -26 \\
\hline
\end{tabular}

es por esto que, para encontrar resultados con estas concentraciones de etanol, fue necesario agregar 7000 y 8000 ppm de agua a la mezcla.

Los resultados de estas pruebas concuerdan con los resultados obtenidos por otros investigadores. Efectivamente, para porcentajes en volumen de etanol de 10\% la separación comienza alrededor de las 4500 ppm de agua y para volúmenes de etanol del $15 \%$, la cantidad límite de agua es superior a los 5000 ppm. Es importante observar que en el trabajo elaborado por Torres et al. [17] se buscaba la concentración de agua para que existiera separación, antes que la temperatura de separación. Los resultados reportados allí coinciden con los reportados en el estudio de Korotney [26] (véase Figura 7).

Sin embargo, los resultados reportados por Kato [18] indican que las temperaturas de separación resultan superiores, lo que representa una menor tolerancia al agua y, por lo tanto, una mayor facilidad de separación. Esto demostraría una menor cantidad de aromáticos y aditivos en la gasolina que se utilizó en ese estudio o eventualmente la influencia de variables como la presión local bajo la que se realizó el ensayo.

\subsection{Efectos de la presión}

Los resultados comparativos de la temperatura de separación entre las muestras E10, E15, E20, y E30 a presión local (75 kPa) y presión de vacío (65 kPa) se observan en la Tabla 5.

El efecto de la presión de vacío es variable. Mientras para algunas concentraciones parece aumentar la temperatura de separación, para otras hace lo contrario. De acuerdo al procedimiento seguido puede establecerse 


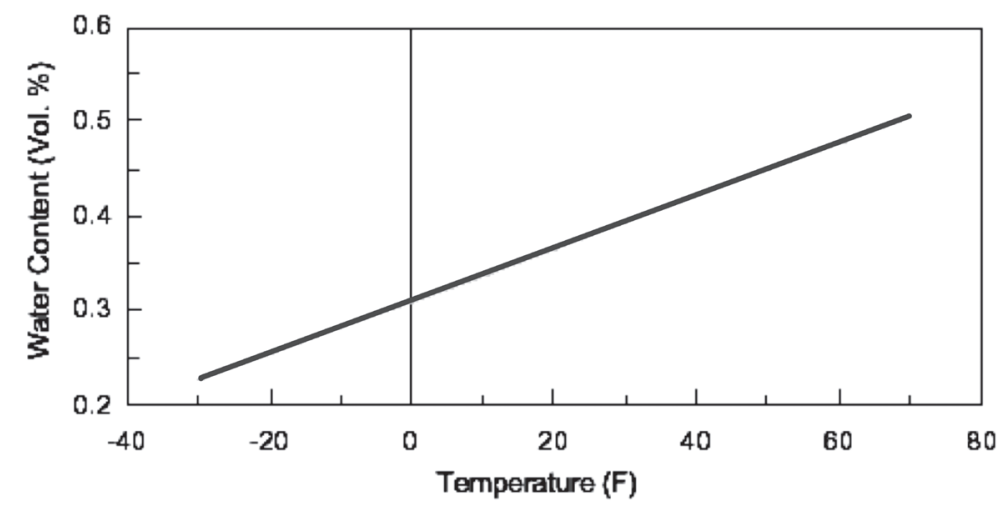

Figura 7. Temperatura de separación para varios contenidos de agua en la mezcla E10.

Fuente: Korotney, 1995.

Tabla 5. Temperaturas de separación de fase de las mezclas evaluadas en dos condiciones de presión.

\begin{tabular}{|c|c|c|c|c|c|c|c|c|}
\hline & \multirow{3}{*}{ Mezcla } & \multicolumn{7}{|c|}{ Temperatura de separación $\left({ }^{\circ} \mathrm{C}\right)$} \\
\hline & & \multicolumn{7}{|c|}{ Contenido de agua (ppm) } \\
\hline & & 3000 & 3500 & 4000 & 4500 & 5000 & 6000 & 7000 \\
\hline \multirow[t]{2}{*}{ E10 } & $\begin{array}{c}\text { A presión } \\
\text { atmosférica } \\
\text { Bogotá (75kPa) }\end{array}$ & -16 & -8 & -6 & $\begin{array}{l}\text { Separación } \\
\text { instantánea }\end{array}$ & $\begin{array}{l}\text { Separación } \\
\text { instantánea }\end{array}$ & & \\
\hline & $\begin{array}{c}\text { A la presión } \\
\text { evaluada }(65 \mathrm{kPa})\end{array}$ & -16 & -6 & -8 & $\begin{array}{l}\text { Separación } \\
\text { instantánea }\end{array}$ & $\begin{array}{l}\text { Separación } \\
\text { instantánea }\end{array}$ & & \\
\hline \multirow{2}{*}{ E15 } & $\begin{array}{c}\text { A presión } \\
\text { atmosférica } \\
\text { Bogotá ( } 75 \mathrm{kPa})\end{array}$ & $\begin{array}{l}\text { No se } \\
\text { encontró }\end{array}$ & -34 & $-17-$ & -16 & -20 & & \\
\hline & $\begin{array}{l}\text { A la presión } \\
\text { evaluada }(65 \mathrm{kPa})\end{array}$ & $\begin{array}{l}\text { No se } \\
\text { encontró }\end{array}$ & -34 & -15 & -14 & -22 & & \\
\hline \multirow{2}{*}{ E20 } & $\begin{array}{c}\text { A presión } \\
\text { atmosférica } \\
\text { Bogotá ( } 75 \mathrm{kPa})\end{array}$ & $\begin{array}{l}\text { No se } \\
\text { encontró }\end{array}$ & $\begin{array}{l}\text { No se } \\
\text { encontró }\end{array}$ & -40 & -38 & -34 & & \\
\hline & $\begin{array}{c}\text { A presión } \\
\text { atmosférica } \\
\text { Bogotá (65kPa) }\end{array}$ & $\begin{array}{l}\text { No se } \\
\text { encontró }\end{array}$ & $\begin{array}{l}\text { No se } \\
\text { encontró }\end{array}$ & & -36 & -34 & & \\
\hline \multirow{2}{*}{ E30 } & $\begin{array}{c}\text { A presión } \\
\text { atmosférica } \\
\text { Bogotá ( } 75 \mathrm{kPa})\end{array}$ & $\begin{array}{l}\text { No se } \\
\text { encontró }\end{array}$ & $\begin{array}{l}\text { No se } \\
\text { encontró }\end{array}$ & $\begin{array}{l}\text { No se } \\
\text { encontró }\end{array}$ & $\begin{array}{l}\text { No se } \\
\text { encontró }\end{array}$ & $\begin{array}{l}\text { No se } \\
\text { encontró }\end{array}$ & -38 & -26 \\
\hline & $\begin{array}{c}\text { A presión } \\
\text { atmosférica } \\
\text { Bogotá (65kPa) }\end{array}$ & $\begin{array}{c}\text { No se } \\
\text { encontró }\end{array}$ & $\begin{array}{l}\text { No se } \\
\text { encontró }\end{array}$ & $\begin{array}{l}\text { No se } \\
\text { encontró }\end{array}$ & $\begin{array}{l}\text { No se } \\
\text { encontró }\end{array}$ & $\begin{array}{l}\text { No se } \\
\text { encontró }\end{array}$ & & -28 \\
\hline
\end{tabular}


que los rangos de observación de la muestra son de dos grados Celsius. Esto quiere decir que hay un margen de error de $\pm 1^{\circ} \mathrm{C}$.

Ahora bien, en ninguna de las pruebas se encuentra diferencia entre las muestras sometidas a vacío y las no sometidas mayor a $2{ }^{\circ} \mathrm{C} \mathrm{y}$, de hecho, algunas de ellas dieron resultados iguales. Esto lleva a concluir que, o bien el efecto de la presión es ligero y depende de las concentraciones, o sencillamente la presión no afecta el comportamiento de las muestras.

\section{CONCLUSIONES}

Los resultados de la gasolina evaluada en este estudio demuestran que la calidad de esta (específicamente en lo referente a la cantidad de aromáticos y aditivos) es inferior a la calidad de la gasolina de la prueba obtenida en Reynolds [19] y, en cambio, superior a la calidad obtenida en Karaosmanoglu [20]. Sin embargo, se reitera que esta afirmación depende de la certeza que se tenga de que las pruebas fueron realizadas bajo el mismo procedimiento.

Por otra parte, la similitud entre los resultados reportados por Torres et al. [17] con los resultados presentados en esta investigación resulta por demás ajustada a la afirmación según la cual ambas pruebas se realizaron con gasolinas similares en sus características, lo que es de hecho cierto porque ambas pruebas se realizan con gasolina colombiana.

En cuanto a la influencia del contenido de etanol y el contenido de agua en la temperatura de separación de la mezcla no se encontró ninguna diferencia con las investigaciones realizadas. Este estudio aporta resultados adicionales para mezclas con gasolinas nacionales E15, E20 y E30 y adicionalmente para E10 a temperaturas menores a los $0^{\circ} \mathrm{C}$.

Sobre el efecto de la presión, tras observar los resultados, se determina que la composición de la mezcla no influye en el efecto que la presión pudiese ocasionar sobre la temperatura de separación, siempre y cuando la mezcla combustible se mantenga en estado líquido. Más aún, se concluye que la presión no afecta el resultado y que las diferencias observadas dependen más de la agitación de la mezcla y de la tolerancia de error que se tiene por la metodología de las pruebas.

\subsection{Recomendaciones}

Uno de los aspectos más ambiguos a la hora de realizar las pruebas es el agitado de la muestra. En general, este procedimiento se debe realizar porque la temperatura en la parte inferior del contenedor llega a un menor valor más rápido que en la parte superior y es necesaria la agitación para homogenizar la mezcla. Si esto no se hiciese, de cualquier modo la parte inferior se mantendría más fría que la superior porque al ser de mayor densidad no habría opción de que la masa fría suba. Pero realizar la agitación también implica mantener la muestra a condiciones ambiente, lo que se traduce en un cambio de temperatura en la muestra e implica un error en la lectura - la necesidad de hacer una doble lectura. Desde luego, el método recomendado para futuras pruebas es el de realizar una doble lectura, la primera al alcanzar la temperatura de referencia y la segunda tras realizar la agitación y la limpieza del contenedor, siendo claro que esta lectura es registrada solo si se observa la separación.

La precisión a la hora de realizar las mezclas es otro aspecto a tener en cuenta. Se recomienda 
el uso de micropipetas para el desarrollo de futuras pruebas.

Debido a que los resultados con la presión de vacío para la mezcla en fase líquida no fueron concluyentes, ya que existe evidencia de separación de la mezcla cuando es introducida en un motor de combustión interna [2-10-27], es recomendable realizar pruebas de mezclas aire-gasolina-etanol-agua en fase gaseosa bajo presiones de vacío.

\section{REFERENCIAS BIBLIOGRÁFICAS}

[1] Chiaramonti, D. (2007). Bioethanol: role and production technologies. En: P. Ranalli (Ed.). Improvement of crop plants for industrial end uses (pp. 209-251). Países Bajos: Springer.

[2] Backgrounder on low level gasoline blends containing ethanol. (2006) Recuperado el 7 de junio de 2007 de http://www.2006fuels.org/ethanol.htm.

[3] Owen, K. y Coley, T. (1990). Automotive fuels handbook. Warrendale, PA, EE.UU: SAE.

[4] Pearson, R., Turner, J., Bell, A., De Goede, S., Woolard, C., y Davy, M. (2014) Isostoichiometric fuel blends: characterisation of physicochemical propierties for mixtures of gasoline, ethanol, methanol and water. En C. Garner (Ed.), Proceedings of the Institution of Mechanical Engineers, Part D: Journal of automobile engineering (pp. 1-29). UK: SAGE.

[5] Ribeiro M., Angnes, L. y Rocha, F. (2013). A simple and fast procedure for in situ determination of water in ethanol fuel.
Journal of the Brazilian Chemical Society, 24(3), 418-422.

[6] Black, F. (1991). An overview of the technical implications of methanol and ethanol as highway motor vehicle fuels. doi: $10.4271 / 912413$.

[7] Thomson, J., Pawel, S. y Wilson, D. (2013). Susceptibility of aluminum alloys to corrosion in simulated fuel blends containing ethanol. Fuel, 111, 592-597.

[8] Birrell, J. (1985). Ethanol as a petrol extender in spark ignition engines. SAE Paper 825026.

[9] Warner-Selph, M. y Harvey, C. (1990). Assessment of unregulated emissions from gasoline oxygenated blends. doi: $10.4271 / 902131$

[10] Orbital Engine Company. (2002). A literature review based assessment on the impacts of a $20 \%$ ethanol gasoline fuel blend on the Australian vehicle fleet. Recuperado en diciembre de 2014 de http://nmma.org/assets/cabinets/ Cabinet103/ethanol-report.pdf.

[11] Haskew, H. (2001). Evaporative emissions effects (permeation) created by ethanol in gasoline. Recuperado en diciembre de 2014 de http://www.arb.ca.gov/fuels/ gasoline/meeting/2001/053101Pmtn. pdf.

[12] Arters, D., Schiferl, E. y Szappanos, G. (2002). Effects of gasoline driveability index, ethanol and intakevalve deposits on engine performance in a dynamometerbased cold start and warmup procedure. SAE Paper 2002-01-1639. 
[13] Unidad de Planeación Minero Energética, (2013). Cadena del petróleo 2013 UPME. Recuperado el 24 de septiembre de 2014 de http://www1.upme.gov.co/ sala-de-prensa/noticias/cadena-depetroleo-2013.

[14] Universidad Tecnológica de Pereira, Ecopetrol S.A. y Ministerio de Minas y Energía. (s.f.). Efecto de la mezcla de combustible E20 en el parque automotor Colombiano. Recuperado el 24 de septiembre de 2014 de http://oab. ambientebogota.gov.co/apc-aa files/57 c59a889ca266ee6533c26f970cb14a/ efecto_E20_en_parque_automotor.pdf

[15] Alcaldía de Bogotá. (2011). Movilidad en cifras 2011. Recuperado el 24 de septiembre de 2014 de http://www. movilidadbogota.gov.co/hiwebx archivos/audio_y_video/boletin $\% 20$ cifras.pdf.

[16] Strus, B., Sobczyńska, A. y Wiśniewski, M. (2008). Solubility of water and association phenomena in gasoline modified with hydrophilic additives and selected surfactants. Fuels, 87(6), 957963.

[17] Torres, J., Molina, D., Pinto, C. y Rueda, F. (2002). Estudio de la mezcla de gasolina con $10 \%$ de etanol anhidro. Evaluación de propiedades fisicoquímicas. Ciencia, Tecnología y Futuro, 2(3), 71-82.

[18] Kato, K. (2006). Out Look for Bio Fuels - a Japanese perspective. Recuperado en diciembre de 2014 de http://www. ee-design.net/topics/2006/20061214_ contents.html.
[19] Reynolds, R. (2002). Fuel Specifications and fuel property issues and their potential impact on the use of ethanol as a transportation fuel. Report to: Downstream Alternative Inc. South Bend, IN, USA. Recuperado en diciembre de 2014 de http://gisceu.net/PDF/U368.pdf.

[20] Karaosmanoglu, F., Isıgıgur, A. y Aksoy, A. (1996). Effects of a New Blending Agent on Ethanol-Gasoline Fuel. Energy Fuels, 10(3), 816-820.

[21] French, R. y Malone, P. (2005), Phase equilibria of ethanol fuel blends. Fluid Phase Equilibria, 228-229, pp. 27-40.

[22] American Society for Testing and Materials. (1999). Test method for water tolerance (phase separation) of gasolinealcohol blends. doi: 10.1520/D6422-99.

[23] Instituto Colombiano de Normas Técnicas. (2005), Petróleo y sus derivados. Gasolinas para motores de combustión interna de encendido por chispa. ICONTEC NTC 1380. Bogotá, Colombia: ICONTEC.

[24] American Society for Testing and Materials. (2005). Standard test method for density, relative density (specific gravity), or API gravity of crude petroleum and liquid petroleum products by hydrometer method. doi: 10.1520/D1298-12B.

[24] American Society for Testing and Materials. (2003). Standard test method for distillation of petroleum products at atmospheric pressure. doi: 10.1520/D0086-12.

[25] American Society for Testing and Materials. (1992). Standard Test Method 
for Determination of Water in Liquid Petroleum Products by Karl Fischer Reagent. doi: 10.1520/D1744.

[26] Korotney, D. (1995). Water Phase Separation in Oxygenated Gasoline. Recuperado el 24 de septiembre de 2014 de http://www.epa.gov/otaq/regs/fuels/ rfg/waterphs.pdf.

[27] Yücesu, H. y Topgül, T. (2006). Effect of ethanol-gasoline blends on engine performance and exhaust emissions in different compression ratios. Applied Thermal Engineering, 26, 2272-2278. 